\title{
P02-164
}

\section{CORTICOBASAL DEGENERATION PRESENTING WITH DEPRESSION AND DYSTONIA: A CASE REPORT}

J. Cerejeira ${ }^{1}$, R. André ${ }^{2}$, P. Batista ${ }^{1}$, P. Carriço ${ }^{1}$, A.M. Ferreira ${ }^{1}$, H. Firmino ${ }^{1}$, C. Januário ${ }^{2}$

${ }^{1}$ Psychiatry, ${ }^{2}$ Neurology, Hospitais Universidade Coimbra, Coimbra, Portugal

Corticobasal degeneration is a rare neurodegenerative disorder affecting both cortex and basal ganglia with clinical and underlying pathological heterogeneity. Although motor features of CBD were emphasized in earlier descriptions psychiatric symptoms, including cognitive impairment and mood disorders, have been consistently reported during the course of the disease. Clinical diagnosis of $\mathrm{CBD}$ is challenging and can be difficult to differentiate from other neuropsychiatric disorders with overlapping features. This can lead to significant underdiagnosis of CBD particularly during its early stages.

We report a case of a 48 year-old female patient presenting with insidious orofacial dystonia co-occurring with depression which remained controlled for five years. Later, while experiencing major psycho-social stress factors, she presented with a rapidly progressive clinical syndrome compatible with the diagnosis of cortico-basal degeneration with severe motor, cognitive and behavioural symptoms, including alien limb phenomenon, nonfluent aphasia and personality changes. Neuropsychological assessment revealed significant frontal lobe dysfunction and SPECT imaging showed asymetrical fronto-parietal hypoperfusion.

This case illustrates the difficulties in the clinical diagnosis of CBD both in early and late stages due to its clinical overlap with mood and movement disorders as well as with Fronto-Temporal Dementia. At the same time, it highlights the influence of psycho-social stress factors in the manifestation of degenerative disorders. 